University of Nebraska - Lincoln

DigitalCommons@University of Nebraska - Lincoln

Gordon Gallup Publications

Research Papers in Physics and Astronomy

July 1978

Representation of $a b$ initio energy surfaces by analytic functions

R.L. Vance

University of Nebraska - Lincoln

Gordon A. Gallup

UNL,ggallup1@unl.edu

Follow this and additional works at: https://digitalcommons.unl.edu/physicsgallup

Part of the Physics Commons

Vance, R.L. and Gallup, Gordon A., "Representation of ab initio energy surfaces by analytic functions" (1978). Gordon Gallup Publications. 40.

https://digitalcommons.unl.edu/physicsgallup/40

This Article is brought to you for free and open access by the Research Papers in Physics and Astronomy at DigitalCommons@University of Nebraska - Lincoln. It has been accepted for inclusion in Gordon Gallup Publications by an authorized administrator of DigitalCommons@University of Nebraska - Lincoln. 


\title{
Representation of ab initio energy surfaces by analytic functions
}

\author{
R. L. Vance and G. A. Gallup \\ Department of Chemistry, University of Nebraska, Lincoln, Nebraska 68588 \\ (Received 22 February 1978)
}

A method for the analytical representation of $a b$ initio energy surface results is described. For a threeatom surface, the potential function is written as a sum of atomic, two-body, and three-body terms. This allows an easy fitting of the whole surface, including the dissociated and united atom limits. The theory is applied to an $\mathrm{H}_{3}$ surface of limited accuracy calculated for these purposes. Comparisons are made, where possible, with more accurate calculations. Extensions to surfaces involving four or more atoms are also discussed.

\section{INTRODUCTION}

The determination from first principles of cross sections and rate constants for atomic and molecular processes requires a knowledge of the molecular potential energy surface over which the system moves, and a completely theoretical approach involves the use of surfaces obtained by $a b$ initio methods. Recently, much progress has been made in developing methods for doing dynamics calculations given a surface, ${ }^{1}$ but many studies have been made with surfaces defined semiempirically. A successful dynamics calculation requires rapid determination of the potential energy (or force) at nearly continuously distributed sets of molecular geometries. $A b$ initio methods do not lend themselves to giving such results, and interpolation or function fitting techniques must usually be used. Two general philosophies of fitting techniques are now developing. The global approach is exemplified by the least-square functions of $\mathrm{Liu}^{2 b}$ and the analytical functions of Truhlar and Kuppermann. ${ }^{3}$

A local approach, used by McLaughlin and Thompson ${ }^{4}$ and Raff and co-workers, ${ }^{5-9}$ gives a fit of either $a b$ initio or semiempirical potential surfaces with multidimensional cubic spline functions. These workers have found that multidimensional spline fitting of $a b$ initio points can serve as a useful means of interpolation. Cubic splines have also found effective use in this laboratory for fitting $\mathrm{H}_{2}-\mathrm{H}_{2}$ potentials. ${ }^{10}$ In spite of these successes, the problem of obtaining analytic representations of $a b$ initio surfaces for use in dynamics studies is still a major road block impeding their use in theoretical kinetics.

This article presents a general method of fitting $a b$ initio surfaces to polynomial expressions. The discussion will concentrate on the reactive surface for three atoms, but we show how extentions to tetra-atomic systems can be made. The pivotal step of our method consists in isolating types of mathematical behavior of the surface in different terms so that they may be dealt with most simply. As shall be seen, the separation of terms providing the isolation also separates apart various physical and chemical phenomena for simpler qualitative understanding.

In many cases, the dynamics calculations require only a portion of the surface. Nevertheless, we examine the problem of fitting the whole range of variables by polynomial expressions.

\section{THE GENERAL MATHEMATICAL PROPERTIES OF AN XYZ SURFACE}

It is useful to examine the properties of a general triatomic surface. The internal geometric configuration is completely determined by the three interatomic distances $r_{12}, r_{23}$, and $r_{13}$, which we use for the variables of the potential functions, $V\left(r_{12}, r_{23}, r_{13}\right)$. From the mathematical point of view, the most striking features of $V$ are the three poles of order 1 when $r_{12}, r_{23}$, or $r_{13}$ becomes zero. Most analytic fitting techniques for complicated functions involve either directly or indirectly the use of approximating polynomials, and it is well known that polynomials cannot represent poles well. Of course, the existance of poles can also have considerable influence at points distant from them, as is shown in the theory of convergence of power series in the complex plane.

These facts suggest that we can ease the polynomial fitting problems by eliminating the poles from $V$. This might be done by subtracting $Z_{1} Z_{2} / r_{12}$ and corresponding terms for the other pairs from $V$. These constitute the exact form of the poles from the nuclear term in the Hamiltonian for an adiabatic surface. The potential function $V$, modified in this way, still retains regions of reasonably rapid fluctuation. One may go farther and subtract other quantities from $V$. The procedure we use consists of writing $V$ as a sum of three kinds of terms, ${ }^{11}$

$$
\begin{aligned}
V\left(r_{12}, r_{23}, r_{13}\right)= & A_{1}+A_{2}+A_{3}+D_{12}\left(r_{12}\right)+D_{23}\left(r_{23}\right) \\
& +D_{13}\left(r_{13}\right)+T_{123}\left(r_{12}, r_{23}, r_{13}\right),
\end{aligned}
$$

where $A_{i}$ is the energy at infinity of the $i$ th atom, $D_{i j}$ is the diatomic molecule potential of the $i-j$ atom pair, and $T_{123}$ is the remainder of the potential. The $D_{i j}$ in (1) must be arranged to be asymptotic to zero for large values of $r_{i j}$. No approximations are involved in (1), of course.

The benefits of writing the energy in this form arise because the large fluctuations in $V$ derive mainly from the $D_{i j}$, which now are functions of only one variable and may be dealt with quite simply. The difficult term, $T_{123}$, is quite smooth, and is zero except in those regions where significant chemical interaction of all three atoms occurs. We return to this aspect of the threebody term for more discussion later. 


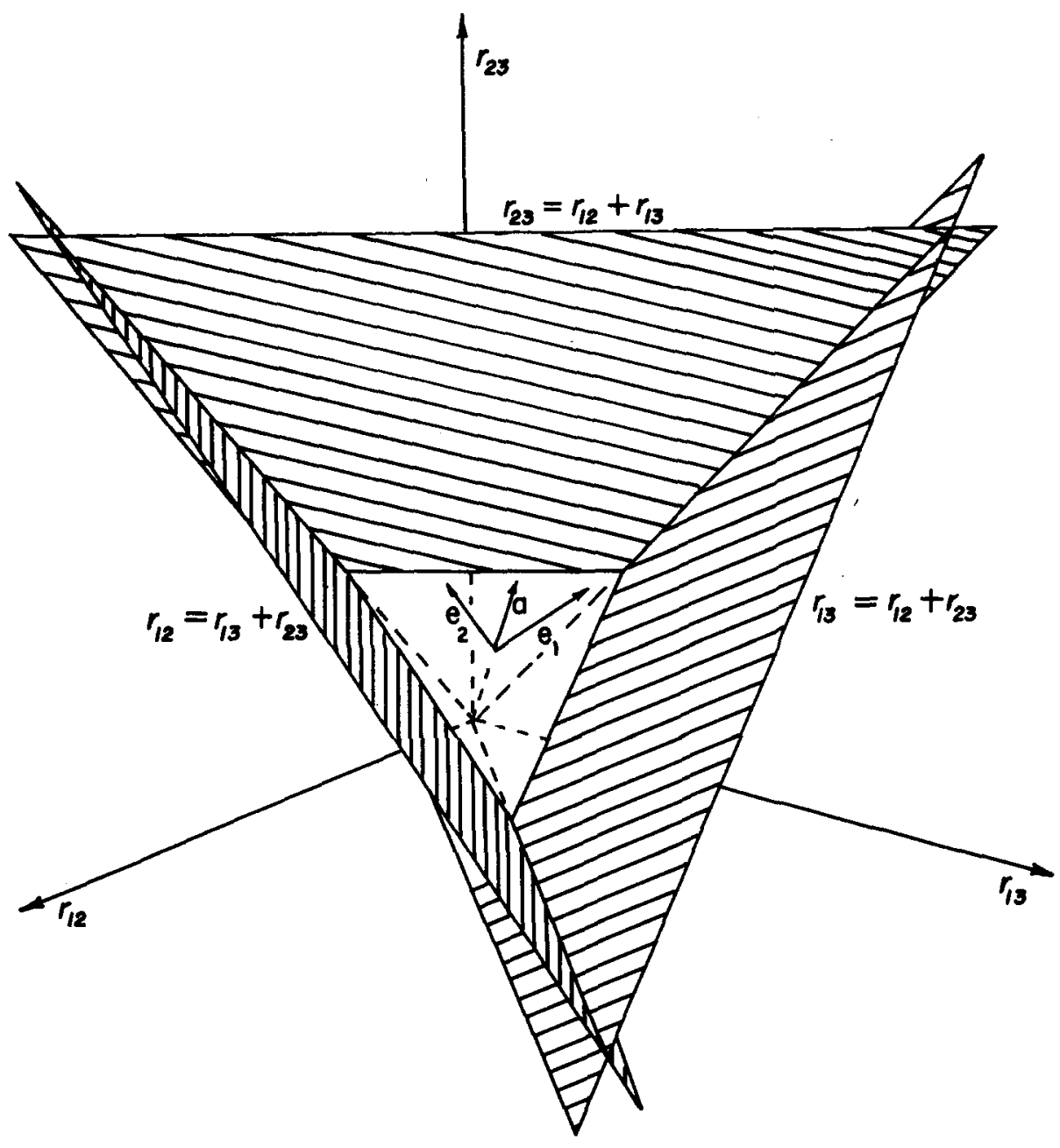

FIG. 1. The $r_{12}, r_{13}, r_{23}$ coordinate system, constraining planes and relations to $a, e_{1}$, and $e_{2}$.

\section{DESCRIPTION OF $T_{123}$}

The use of cubic splines and piecewise-Hermite polynomials ${ }^{12}$ for representing arbitrary functions has become popular in recent years. ${ }^{13}$ Multidimensional applications are possible and we use a combination of bicubic Hermite interpolation and cubic splines.

$T_{123}$ is a function of the three coordinates $r_{12}, r_{13}, r_{23}$. We consider them to be a Cartesian set, but the values for the physical system are subject to restrictions,

$$
\begin{aligned}
& r_{12} \geq 0, \\
& r_{23} \geq 0, \\
& r_{13} \geq 0, \\
& r_{12}+r_{23} \geq r_{13}, \\
& r_{29}+r_{13} \geq r_{12}, \\
& r_{13}+r_{12} \geq r_{23} .
\end{aligned}
$$

The last three inequalities of (2) define planes in the $r_{12}, r_{13}, r_{23}$ space which collectively confine the system point to a portion of the first octant as shown in Fig. 1. $r_{12}, r_{13}$, and $r_{23}$ are not themselves a convenient set of coordinates to use, and we transform to a new set,

$$
a=\left(r_{12}+r_{13}+r_{23}\right) / 3 \text {, }
$$

$$
\begin{aligned}
& e_{1}^{\prime}=2\left(r_{13}+r_{23}-2 r_{12}\right) / 3 \sqrt{3} \\
& e_{2}^{\prime}=2\left(r_{23}-r_{13}\right) / 3
\end{aligned}
$$

The new coordinate $a$ is subject to the restriction $a \geq 0$, only, and the $e_{1}^{\prime}-e_{2}^{\prime}$ coordinates satisfy the inequalities

$$
\begin{aligned}
& e_{1}^{\prime} \geq-a / \sqrt{3}, \\
& e_{2}^{\prime} \geq e_{1}^{\prime} / \sqrt{3}-2 a / 3, \\
& e_{2}^{\prime} \geq e_{1}^{\prime} / \sqrt{3}+2 a / 3,
\end{aligned}
$$

which define an equilateral triangle in the $e_{1}^{\prime}-e_{2}^{\prime}$ plane as shown in Fig. 2.

A simple rotation would yield new coordinates in which $a$ is "stretched" by a factor $\sqrt{3}$ compared to (3). Our choice makes $a$ the arithmetic mean of $r_{12}, r_{13}$, and $r_{23}$, and gives a "better feel" for the size of the system. The coordinates $e_{1}^{\prime}$ and $e_{2}^{\prime}$ are subjected to a further stretching so that $-1 \leq e_{2}^{\prime} / a \leq 1$, which provides some later simplifications.

A triatomic system may have the form $X Y Z, X_{2} Y$, or $\mathrm{X}_{3}$. In the latter two cases $T_{123}$ possesses symmetry, and the coordinates $a, e_{1}^{\prime}$, and $e_{2}^{\prime}$ lie parallel or perpendicular to the symmetry planes. The resultant simplifications constitute the main justification for their use. 


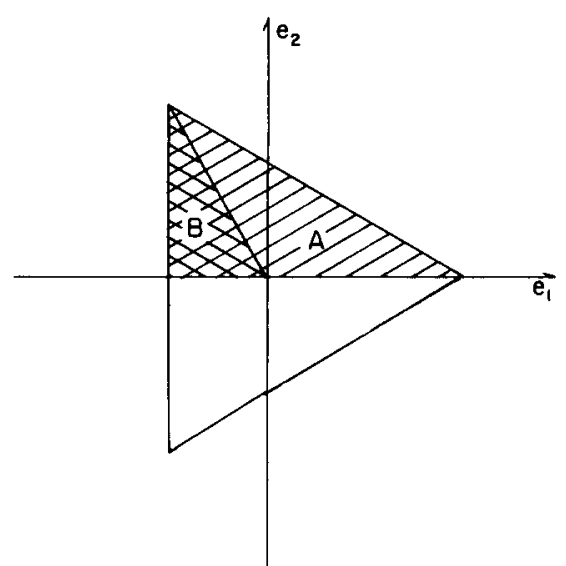

FIG. 2. The primary areas of calculation for $X_{2} Y, A+B$; and for $\mathrm{X}_{3}, \mathrm{~B}$.

\section{A. $X_{2} Y$ case}

Let us take atoms 1 and 2 to be the same and atom 3 different. Under these circumstances, the potential $V$ and also $T_{123}$ are invariant to a permutation of the atom labels 1 and 2. This can be viewed as a reflection in the $a-e_{1}^{\prime}$ plane, or the values of $T_{123}$ in the upper-half triangle in Fig. 2 are reflected into the lower half. Therefore, when obtaining the original energies and represent. ing them, we need only to know half of the triangle.

\section{B. $\mathrm{X}_{3}$ case}

When all three atoms are the same, we need determine $T_{123}$ in only one-sixth of the triangle. The functions $V$ and $T_{123}$ are invariant to all six permutations of the atom labels, or the symmetric group, $S_{3}$. These operations on the labels generate the operations of the point group $C_{3 v}$ (which is isomorphic to $S_{3}$ ) in the $a, e_{1}^{\prime}$, $e_{2}^{\prime}$ space. The $C_{3}$ axis is along $a$, of course. Once we know the values of $T_{123}$ in one of the small triangles in Fig. 2, we may rotate and reflect these values around to obtain the function everywhere.

When it is present, the symmetry provides other useful information for the fitting procedure. The gradient of $T_{123}$ must be zero on and perpendicular to a symmetry plane. As we shall see, such symmetry conditions on the gradient help to determine the functions we use for representing $T_{123}$.

\section{REPRESENTATION OF $T_{123}$ BY TRICUBIC POLYNOMIALS}

The triangular pyramid region of Fig. 1 is still not completely suitable for tricubic polynomial fitting, and one more modification is necessary. We will actually use $e_{1}=e_{1}^{\prime} / a$ and $e_{2}=e_{2}^{\prime} / a$ in our expressions. The original set would require either of two unworkable alternatives, i.e., increasing the number of regions as $a$ increases, or fitting regions bounded by surfaces none of which meet at right angles.

With this further transformation we will apply our theory to the $\mathrm{X}_{3}$ case. Extensions to less symmetric systems are easily made.
Figure 3 shows the small triangle which we take as our primary fitting region. This may be divided into a number of rectangular and triangular regions. A tricubic polynomial is written

$$
P^{(3)}\left(a, e_{1}, e_{2}\right)=\sum_{i=0}^{3} \sum_{j=0}^{3} \sum_{k=0}^{3} C_{i j k} a^{i} e_{1}^{j} e_{2}^{k},
$$

and 64 coefficients are required to define the function in one right angled parallelepiped shaped volume. One set is needed for each of the regions we take in the $a, e_{1}$, and $e_{2}$ directions. In addition to the full regions, we also have the ones shaped like triangular prisms. Again referring to $\mathrm{Fig}$. 3 , the symmetry requires the gradient of $T_{123}$ to be zero across the lines $\overline{A O}$ and $\overrightarrow{B O}$. Such requirements reduce the number of independent coefficients in (5) to 40. A reasonably simple calculation shows that this reduction a rises from the 24 relations,

$$
\begin{aligned}
& \sqrt{3} a_{i 10}+a_{i 01}=0, \\
& \sqrt{3} a_{i 20}-a_{i 11}-\sqrt{3} a_{i 02}=0, \\
& 3 \sqrt{3} a_{i 30}-5 a_{i 21}+\sqrt{3} a_{i 12}+9 a_{i 03}=0, \\
& 2 a_{i 31}-\sqrt{3} a_{i 22}=0, \\
& 7 \sqrt{3} a_{i 32}-9 a_{i 23}=0, \\
& a_{i 33}=0,
\end{aligned}
$$

in which $i$ takes on the values $0,1,2$, and 3 . When these relations are used with the standard connections at the knots, or with known slopes at each knot, we have a set of tricubic polynomials to represent $T_{123}$ in the primary triangle, and throughout the whole region.

\section{APPLICATION AND DISCUSSION}

It is not the purpose of this article to present an energy surface of high accuracy for the $\mathrm{H}_{3}$ system. We have however, calculated the energies of $\mathrm{H}_{3}$ with a [2s] basis at a number of points in the primary triangle, and applied the theory of the previous sections to test these procedures. We have purposely used a very small number of geometries in our calculations. This will make the test severe. Unfortunately, earlier, more accurate calculations, ${ }^{2}$ have not been done at a set of geometries appropriate to the present treatment.

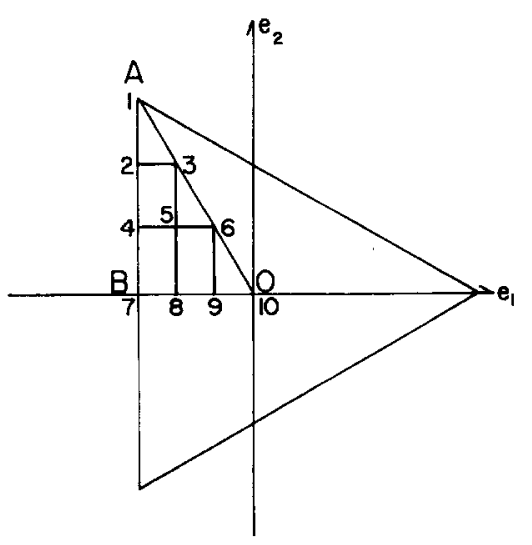

FIG. 3. The mesh of points for calculating 10 point energy surface for $\mathrm{X}_{3}$. 


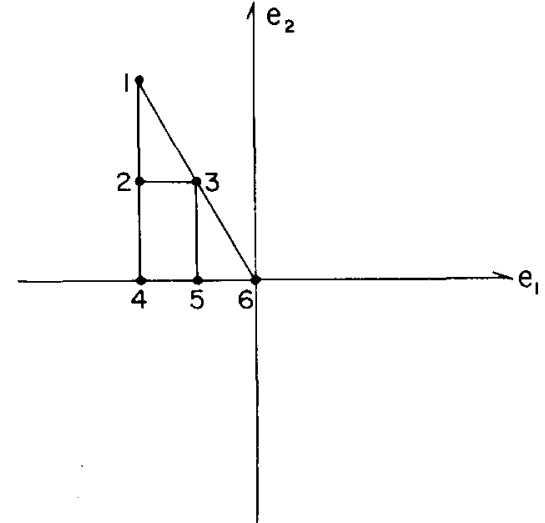

FIG. 4. Positions used in primary triangle for present $\mathrm{H}_{3}$ calculation.

A full CI calculation based upon the six-function basis, with a $1 s$ and a $1 s^{\prime}$ at each nuclear center, was done at 36 geometries. Figure 4 shows the positions calculated in the primary triangle, and Table I gives the actual values of $r_{12}, r_{13}$, and $r_{23}$ used. An energy of $\mathrm{H}_{2}$ with the same basis was also determined at each of the $r_{i j}$ values present in the $\mathrm{H}_{3}$ calculation. Our results give a dissociation energy for $\mathrm{H}_{2}$ of $98 \mathrm{kcal}$, approximately 11 kcal too low. In spite of its deficiencies, the $\mathrm{H}_{3}$ calculation reported here describes the important physical aspects of the real system. In several cases, additional CI calculations beyond the original 36 were done for comparisons.

Figure 5 is a three-dimensional contour drawing which shows graphically how $T_{123}$ behaves as a function of $a$, $e_{1}$, and $e_{2}$. The important property of smoothness is apparent. We also see that $T_{123}$ is in general a monotonically decreasing function of $a$. We use this fact and will actually fit $e^{\alpha a} T_{123}$ with our tricubic polynomials. Each of the three regions shown in Fig 4 will be fit with a bicubic Hermite polynomial for the averages $a=1,2,3,4,6$. We then consider the coefficients for the above polynomials to be functions of $a$ and we fit the coefficients with cubic splines. The full tricubic polynomial is written

$$
\begin{gathered}
T_{123}^{l m n}\left(a, e_{1}, e_{2}\right)=e^{-\alpha a} \sum_{i=0}^{3} \sum_{j=0}^{3} \sum_{k=0}^{3} c_{i j k}^{l m n}\left(e_{1}-e_{1, m}\right)\left(e_{2}-e_{2, n}\right)\left(a-a_{l}\right) \\
\text { for } \alpha=0.5, e_{1, m} \leq e_{1} \leq e_{1, m+1}, \\
e_{2, n} \leq e_{2} \leq e_{2, n+1}, \text { and } a_{l} \leq a \leq a_{l+1} .
\end{gathered}
$$

The quantities, $a_{l}, e_{1, m}$, and $e_{2, n}$ are the boundaries of the interpolation regions for the tricubic polynomials. The ground state electronic energy of the $\mathrm{H}_{2}$ molecule, as a function of the internuclear distance $R$, is represented as a piecewise-cubic spline. The authors will be happy to provide anyone with actual coefficients for calculating the $\mathrm{H}_{3}$ surface described here. The region from 0.0 to 6.0 has been divided into 11 regions and the coefficients for a cubic spline determined in each. The spline is written

$$
E_{\mathrm{H}_{2}}^{1}(R)=\sum_{j=0}^{3} C_{j, i}\left(R-R_{i}\right)^{j}
$$

\begin{tabular}{|c|c|c|c|c|c|}
\hline Point ${ }^{\mathrm{a}}$ & $a$ & $r_{12}$ & $r_{13}$ & $r_{23}$ & $T_{123}$ \\
\hline \multirow[t]{5}{*}{1} & 1 & $1.500^{\mathrm{b}}$ & 0.000 & 1.500 & 0.479480 \\
\hline & 2 & 3.000 & 0.000 & 3.000 & 0.113526 \\
\hline & 3 & 4.500 & 0.000 & 4.500 & 0.013572 \\
\hline & 4 & 6.000 & 0.000 & 6.000 & 0.001162 \\
\hline & 6 & 9.000 & 0.000 & 9.000 & 0.000006 \\
\hline \multirow[t]{5}{*}{2} & 1 & 1.500 & 0.375 & 1.125 & 0.538096 \\
\hline & 2 & 3.000 & 0.750 & 2.250 & 0.185144 \\
\hline & 3 & 4.500 & 1.125 & 3.375 & 0.042945 \\
\hline & 4 & 6.000 & 1.500 & 4.500 & 0.007592 \\
\hline & 6 & 9.000 & 2.250 & 6.750 & 0.000167 \\
\hline \multirow[t]{5}{*}{3} & 1 & 1.250 & 0.500 & 1.250 & 0.583582 \\
\hline & 2 & 2.500 & 1.000 & 2.500 & 0.209033 \\
\hline & 3 & 3.750 & 1.500 & 3.750 & 0.046241 \\
\hline & 4 & 5.000 & 2.000 & 5.000 & 0.007122 \\
\hline & 6 & 7.500 & 3.000 & 7.500 & 0.000104 \\
\hline \multirow[t]{5}{*}{4} & 1 & 1.500 & 0.750 & 0.750 & 0.575357 \\
\hline & 2 & 3.000 & 1.500 & 1.500 & 0.247013 \\
\hline & 3 & 4.500 & 2.250 & 2.250 & 0.098955 \\
\hline & 4 & 6.000 & 3.000 & 3.000 & 0.040169 \\
\hline & 6 & 9.000 & 4.500 & 4.500 & 0.004885 \\
\hline \multirow[t]{5}{*}{5} & 1 & 1.250 & 0.875 & 0.875 & 0.648216 \\
\hline & 2 & 2.500 & 1.750 & 1.750 & 0.284748 \\
\hline & 3 & 3.750 & 2.625 & 2.625 & 0.096254 \\
\hline & 4 & 5.000 & 3.500 & 3.500 & 0.027131 \\
\hline & 6 & 7.500 & 5.250 & 5.250 & 0.001594 \\
\hline \multirow[t]{5}{*}{6} & 1 & 1.000 & 1.000 & 1.000 & 0.735297 \\
\hline & 2 & 2.000 & 2.000 & 2.000 & 0.355576 \\
\hline & 3 & 3.000 & 3.000 & 3.000 & 0.129346 \\
\hline & 4 & 4.000 & 4.000 & 4.000 & 0.033873 \\
\hline & 6 & 6.000 & 6.000 & 6.000 & 0.001351 \\
\hline
\end{tabular}

TABLE I. Geometries and energies of three-body terms for $\mathrm{H}_{3}$ calculation.

a This refers to the point numbering in Fig. 4.

${ }^{b}$ All distances and energies are in atomic units.

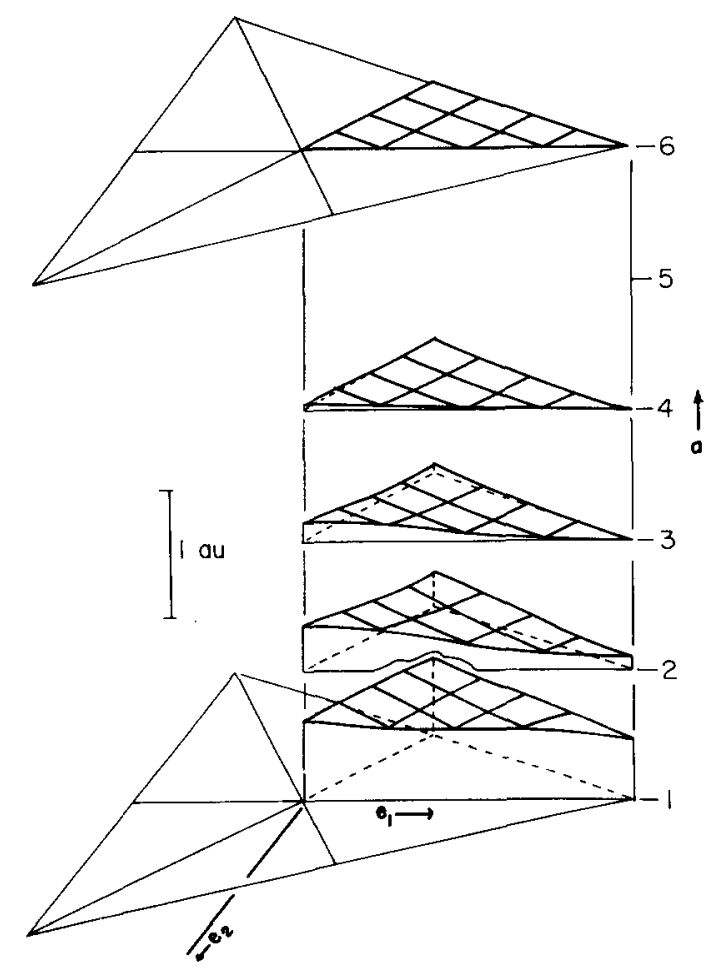

FIG. 5. Graphical representations of $T_{123}$ for $\mathrm{H}_{3}$. 
TABLE II. Potential-energy surface properties in saddle point region.

\begin{tabular}{lccccc}
\hline \hline Surface & $R_{s p}$ & $E_{s p}{ }^{\mathrm{a}}$ & $A_{11}$ & $A_{22}$ & $A_{33}$ \\
\hline 1s, 1s' & 1.791 & -1.6248 & 0.276 & 0.029 & -0.130 \\
Analytical fit & 1.786 & -1.6270 & 0.351 & 0.030 & -0.100 \\
Shavitt et al. ${ }^{\mathrm{c}}$ & 1.764 & -1.6521 & 0.31 & 0.024 & -0.061 \\
Liu $^{\mathrm{d}}$ & 1.757 & -1.6581 & 0.32 & $\ldots$ & -0.058 \\
\hline \hline
\end{tabular}

${ }^{a} E_{s p}$ is the total energy at the saddle point.

bll quantities are expressed in atomic units.

'Reference 2(a)。

${ }^{\mathrm{d}}$ Reference $2(\mathrm{~b})$.

for $R_{i} \leq R \leq R_{i+1}$. The region 6.0 to $\infty$ is represented by a simple inverse power of $R$.

We may use our surface for the determination of the saddle point geometry and the force constants at the saddle point. The saddle point is defined as the minimum in the potential energy curve for linear symmetric $\mathrm{H}_{3}$. The separation at this point, $R_{s p}$, is defined by the following condition:

$$
\left.\frac{\partial}{\partial R} V_{\mathrm{H}_{3}}\left(R_{\mathrm{AB}}, R_{\mathrm{BC}}\right)\right|_{R_{\mathrm{AB}}=R_{\mathrm{BC}}=R_{\mathrm{Sp}}}=0,
$$

with $R_{\mathrm{AB}}$ and $R_{\mathrm{BC}}$ the interparticle distances. The force constants $A_{i i}$ are defined in terms of the normal coordinates for the saddle point configuration of $\mathrm{H}_{3}$, where

$$
\begin{aligned}
& g_{1}=1 / 2\left(R_{\mathrm{AB}}+R_{\mathrm{BC}}-2 R_{\mathrm{sD}}\right), \\
& g_{2}=1 / 2 R_{\mathrm{sD}}(\pi-\theta), \\
& g_{3}=1 / 2\left(R_{\mathrm{AB}}-R_{\mathrm{BC}}\right),
\end{aligned}
$$

with $\theta$ being the angle $\angle \mathrm{ABC}$. The force constants are then

$$
\begin{aligned}
& A_{11}=\left.\frac{\partial^{2} V}{\partial g_{1}^{2}}\right|_{\delta_{1}=\varepsilon_{2}=\varepsilon_{3}=0}, \\
& A_{22}=\left.\frac{\partial^{2} V}{\partial g_{2}^{2}}\right|_{\delta_{1}=\varepsilon_{2}=8 \varepsilon_{3}=0}, \\
& A_{33}=\left.\frac{\partial^{2} V}{\partial g_{3}^{2}}\right|_{\delta_{1}=\varepsilon_{2}=\delta_{3}=0} .
\end{aligned}
$$

Values for the force constant determined from $V$ as well as values from previous calculations are given in Table II.

In a recent calculation Norbeck and Certain ${ }^{14}$ found the triangular arrangement of $\mathrm{H}_{3}$ to be favored over the linear arrangement for values of $R>3.1 a_{0}$ (see Fig. 1, Ref. 14). Results from our polynomial surface and our $a b$ initio surface are compared with those in Fig. 6.

It is evident that properties calculated with the polynomial surface do not agree completely with those calculated using the $a b$ initio surface. However, the qualitative agreement is excellent. This is especially en-

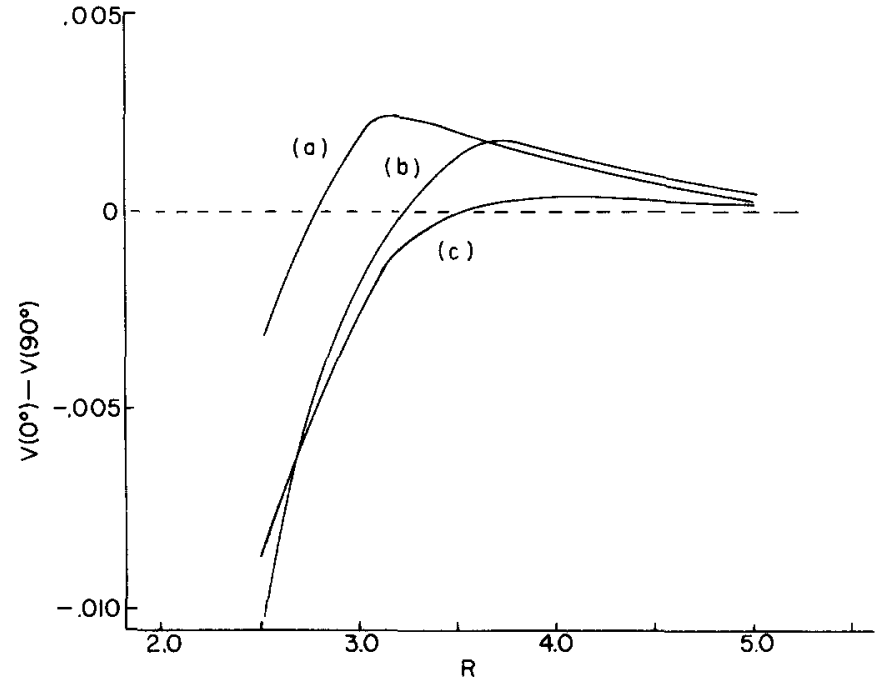

FIG. 6. Stability of the triangular arrangement of $\mathrm{H}_{2}+\mathrm{H}$. Curve (a), 1s GTO (Table I, Ref. 14); Curve (b), Eq. (7), Table II; Curve (c), [2s] basis, this work. $\theta$ and $R$ refer, respectively, to Fig. 1, Ref. 14 .

couraging in light of the low number of energy points used in determining the polynomials. We feel that the arrangement of geometries described here gives the greatest efficiency of utilization of $a b$ initio energies for polynomial fitting. Of course, our technique does not need to be applied to the entire surface, but may be restricted if only some regions are of interest.

The tricubic polynomial representation of a surface requires a large number of coefficients. This can be looked upon as the result of precalculating and storing intermediate results in the interpolation problem. When the tricubic polynomial representation of a surface is used in a computer, an efficient algorithm for finding one polynomial is needed. With equal spacing on the regions as we have used, this algorithm can be designed so that the time required is independent of the total number of regions. Then a very important property of the tricubic polynomial representation becomes evident: The time required on the computer to retrieve a value of $V$ is independent of the accuracy of the calculation, which affects only the storage required. No global method of function fitting will have this property.

\section{EXTENSIONS TO SURFACES INVOLVING MORE THAN THREE ATOMS}

\section{A. Four-atom surfaces}

The geometric representation for three-atom surfaces described in Sec. II may be extended to the case of four atoms. Here again, we have written the general potential in terms of two-, three-, and four-body functions,

$$
\begin{aligned}
& V\left(r_{12}, r_{13}, r_{14}, r_{23}, r_{24}, r_{34}\right) \\
&=A_{1}+A_{2}+A_{3}+A_{4}+D_{12}\left(r_{12}\right)+D_{13}\left(r_{13}\right)+D_{14}\left(r_{14}\right)+D_{23}\left(r_{23}\right)+D_{24}\left(r_{24}\right)+D_{34}\left(r_{34}\right)+T_{123}\left(r_{12}, r_{23}, r_{13}\right) \\
&+T_{124}\left(r_{12}, r_{14}, r_{24}\right)+T_{134}\left(r_{13}, r_{14}, r_{34}\right)+T_{234}\left(r_{23}, r_{24}, r_{34}\right)+Q_{1234}\left(r_{12}, r_{13}, r_{14}, r_{23}, r_{24}, r_{34}\right)
\end{aligned}
$$


As in (1), the poles are all contained in the two-body terms.

The two- and three-body terms in (12) may be handled the way we have shown before. The four-body term presents a new problem. In this case, it is again convenient to make a transformation of variables,

$$
\begin{aligned}
& a=\left(r_{12}+r_{13}+r_{14}+r_{23}+r_{24}+r_{34}\right) / \sqrt{6}, \\
& e_{1}=\left(2 r_{12}+2 r_{34}-r_{13}-r_{14}-r_{23}-r_{24}\right) / \sqrt{12}, \\
& e_{2}=\left(r_{13}+r_{24}-r_{14}-r_{23}\right) / 2, \\
& t_{1}=\left(r_{12}-r_{34}\right) / \sqrt{2}, \\
& t_{2}=\left(r_{13}-r_{24}\right) / \sqrt{2}, \\
& t_{3}=\left(r_{14}-r_{23}\right) / \sqrt{2},
\end{aligned}
$$

which form bases for the $[4],[3,1]$, and $[2,2]$ representations of the symmetric group, $S_{4}$. There are 12 inequalities of the type $r_{i j}+r_{j h} \geq \gamma_{i k}$, plus the four tetrahedral inequalities involving the areas of the four faces, $A_{i}+A_{j}+A_{k}-A_{l} \geq 0$, etc. , which must be satisfied. These constrain $e_{1}$ and $e_{2}$ to a triangular shaped region as before, and $t_{1}, t_{2}$, and $t_{3}$ to a tetrahedrally shaped volume.

A hexacubic polynomial to represent $Q_{1234}$ in a sixdimensional volume requires $4^{6}=4096$ coefficients. This number, although large, is probably not out of the question for the computer technology on the horizon. The results of the three-atom suggest that $Q_{1234}$ might be smoother than even the $T_{123}$-type terms. Concrete $a b$ initio results to test this do not now exist.

\section{B. Many-atom surfaces}

The previous results appear to allow generalization, but the representation of spline functions for five or more centers will require prodigious numbers of coefficients. We shall not consider these cases further.

\section{ACKNOWLEDGMENTS}

The authors wish to express their sincere appreciation to the National Science Foundation for the support of this work through CHE76-17567. We also wish to thank the Computing Network of the University of Nebraska for a grant of computing time.

${ }^{1}$ R. P. Saxon and J. C. Light, J. Chem. Phys. 56, 3874, 3885 (1972); D. L. Bunker, Meth. Comp. Phys. 10, 287 (1971); D. A. Micha, Adv. Chem. Phys. 30, 7 (1975); W. H. Miller, ibid. 30, 77 1975); See announcement, Science 191, 357 (1976).

${ }^{2}$ (a) I. Shavitt, R. M. Stevens, F. L. Minn, and M. Karplur, J. Chem. Phys. 48, 2700 (1968); (b) B. Liu, J. Chem. Phys. 58, 1925 (1973).

${ }^{3}$ D. G. Truhlar and A. Kuppermann, J. Chem. Soc. 93, 1840 (1971).

${ }^{4}$ D. R. McLaughlin and D. L. Thompson, J. Chem. Phys. 59, 4393 (1973).

${ }^{5}$ N. Sathyamurthy, J. W. Duff, C. Stroud, and L. M. Raff, J. Chem. Phys. 67, 3563 (1977).

${ }^{6}$ C. Stroud, N. Sathyamurthy, R. Rangarajan, and L. M. Raff, Chem. Phys. Lett. 48, 350 (1977).

${ }^{7}$ N. Sathyamurthy, R. Rangarajan, and L. M. Raff, J. Chem. Phys. 64, 4606 (1976).

${ }^{8}$ N. Sathyamurthy, G. E. Kellerhals, and L. M. Raff, J. Chem. Phys. 64, 2259 (1976).

${ }^{9}$ N. Sathyamurthy and L. M. Raff, J. Chem. Phys. 63, 464 (1975).

${ }^{10}$ G. A. Gallup, Mol. Phys. 33, 943 (1977).

${ }^{11} \mathrm{~J}$. N. Murrell and S. Farnatos, Mol. Phys. 34, 1185 (1977); S. Farantos, E. C. Leisegang, J. N. Murrell, K. Sorbie, J. J. C. Texeira-Dias, and A. J. C. Varandas, Mol. Phys. 34, 947 (1977); J. N. Murrell, K. S. Sorbie, and A. J. C. Varandas, Mol. Phys. 32, 1359 (1976); K. S. Sorbie and J. N. Murrell, Mol. Phys. 29, 1387 (1975).

${ }^{12}$ S. D. Conte and C. de Boor, Elementary Numerical Analysis: An Algorithmic Approach (McGraw-Hill, New York, 1965).

${ }^{13}$ W. J. Whiten, Australian Comp. J. 3, 81 (1971); C. de Boor, J. Math. Phys, 41, 212 (1962); C. A. Hall, J. Math. Mech. 19, 1 (1969); see also Refs. 4 and 9.

${ }^{14} \mathrm{~J}$. M. Norbeck and P. R. Certain, J. Chem. Phys. 63, 4127 (1975). 